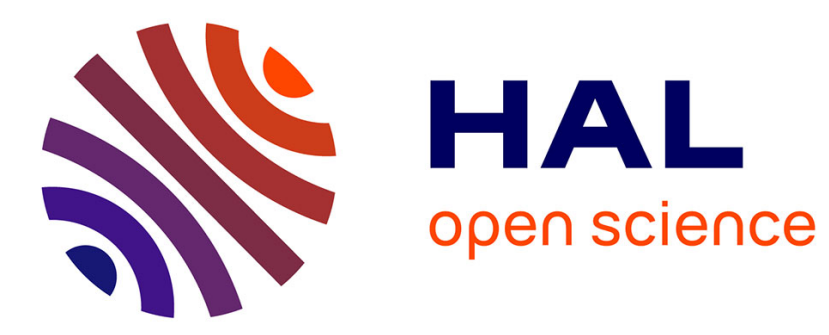

\title{
Heterostructures of two-dimensional transition metal dichalcogenides: formation, ab initio modelling and possible applications
}

A.V. V Krivosheeva, V.L. L Shaposhnikov, V.E. E Borisenko, J.-L. Lazzari

\section{- To cite this version:}

A.V. V Krivosheeva, V.L. L Shaposhnikov, V.E. E Borisenko, J.-L. Lazzari. Heterostructures of twodimensional transition metal dichalcogenides: formation, ab initio modelling and possible applications. 17th International Conference on Nanosciences \& Nanotechnologies (NN20), Nanotexnology, Jul 2020, Thessaloniki, Greece. 10.1016/j.matpr.2021.10.217 . hal-03451177

\author{
HAL Id: hal-03451177 \\ https://hal.science/hal-03451177
}

Submitted on 26 Nov 2021

HAL is a multi-disciplinary open access archive for the deposit and dissemination of scientific research documents, whether they are published or not. The documents may come from teaching and research institutions in France or abroad, or from public or private research centers.
L'archive ouverte pluridisciplinaire HAL, est destinée au dépôt et à la diffusion de documents scientifiques de niveau recherche, publiés ou non, émanant des établissements d'enseignement et de recherche français ou étrangers, des laboratoires publics ou privés. 


\title{
Heterostructures of two-dimensional transition metal dichalcogenides: formation, ab initio modelling and possible applications
}

\author{
A.V. Krivosheeva ${ }^{\mathrm{a} 1}$, V.L. Shaposhnikova ${ }^{\mathrm{a}}$, V.E. Borisenko ${ }^{\mathrm{a}, \mathrm{b}}$, J.-L. Lazzari ${ }^{\mathrm{c}}$ \\ ${ }^{a}$ Belarusian State University of Informatics and Radioelectronics, P. Browka 6, 220013 Minsk, Belarus \\ ${ }^{b}$ National Research Nuclear University MEPhI, Kashirskoe Shosse 31, Moscow, 115409, Russia \\ ${ }^{c}$ Aix Marseille Univ, CNRS, CINaM, UMR 7325, Case 913, Campus de Luminy 13288, Marseille, France
}

\begin{abstract}
State-of-the-art technologies of fabrication of transition metal dichalcogenide monolayers like $\mathrm{MeX}_{2}$, where $\mathrm{Me}=\mathrm{Mo}, \mathrm{W} ; \mathrm{X}=\mathrm{S}$, $\mathrm{Se}, \mathrm{Te}$, and their based heterostructures are considered. Results of theoretical modeling are analyzed and possibilities of band gap engineering by means of strains, impurities, vacancies, various layer stacking and combination of different materials are presented. It is shown that vacancies and impurities in the positions of metal atoms may drastically change the band gap, even leading to an appearance of metallic properties, whereas a substitution of chalcogen atoms by isovalent atoms changes the properties not so dramatically. Possible applications of heterostuctures with tunable band gaps for creation of transistors, light-emitting diodes, photoelectrochemical cells or photovoltaic devices are proposed and their advantages in comparison with commonly used analogues are discussed.
\end{abstract}

Keywords: dichalcogenide, monolayer, heterostructure, electronic properties, impurity.

\section{Introduction}

Monolayers of transition metal dichalcogenides (TMDs) present a family of low-dimensional structures with many attractive properties. They are characterized by a strong bonding in the crystal plane and weak van-der-Waals forces between neighboring atomic planes. Their individual monolayers, contrary to the bulk counterparts, are preferably direct band gap semiconductors. They are stable and can be combined together with graphene or h-BN within devices of new types. They were employed successfully in the fabrication of low-power field-effect transistors, logic circuits, and phototransistors due to band gap lying in the red to near-infrared range, which is advantageous for light-harvesting and light-detecting applications [1].

In order to enlarge the application area, the band-structure engineering and properties manipulation are needed. This purpose is attainable by means of different ways: application of tensile or compressive strains, doping, introducing of vacancies, etc. As a result magnetic ordering can also arise. The strain-induced magnetism was observed in single-layer $\mathrm{MoS}_{2}$ with atomic single vacancies [2], when spontaneous magnetization under certain biaxial tensile strain occurs due to delocalized electrons of Molybdenum. Magnetic moment of zigzag-edged $\mathrm{MoS}_{2}$ nanoribbons can be largely tuned by uniaxial strains along the nanoribbon growth direction. This fact opens opportunity to design magnetic-switching or logic devices. It is reported that single crystals of $\mathrm{MoS}_{2}$ display ferromagnetism superimposed onto large temperature-dependent diamagnetism and ferromagnetism persisting from $10 \mathrm{~K}$ up to room temperature [3]. An existence of ferromagnetism in this case was explained partly by the presence of zigzag edges in the magnetic ground state at the grain boundaries.

First-principle calculations demonstrate possibilities of chemical absorption of H-, B-, C-, N-, O-, and F- atoms on the surface of two-dimensional (2D) $\mathrm{MoS}_{2}$ substrate to induce magnetic moments in the range of $1.0-2.0 \mu_{\mathrm{B}}$ per $4 \times 4$ supercell [4]. Theoretical investigations on the stability and electronic and magnetic properties of $\mathrm{MoS}_{2}$ nanoribbons with different edges show ferromagnetic and metallic behavior of zigzag $\mathrm{MoS}_{2}$ nanoribbons, whereas armchair nanoribbons are nonmagnetic and semiconducting. Moreover, zigzag nanoribbons are more stable than armchair ones. Both zigzag and armchair $\mathrm{MoS}_{2}$ nanoribbons are more stable than the experimentally available $\mathrm{MoS}_{2}$ nanoclusters. Thus, $\mathrm{MoS}_{2}$ nanoribbons provide many new opportunities in various fields such as nanoelectronics, nanotribology,

${ }^{1}$ Corresponding author. Tel.: +375-17-293-88-69

E-mail address: krivosheeva@bsuir.by 
and catalysis [5]. Manganese dopants substituting Mo sites lead to ferromagnetic coupling via a double-exchange mechanism [6]. The study demonstrates a potential for engineering a new class of atomically thin dilute magnetic semiconductors based on Mn-doped $\mathrm{MoS}_{2}$ monolayers.

Application of strains opens a possibility for strain-dependent electronics. In comparison to their bulk counterparts, 2D materials can sustain much higher elastic strain at which band gaps and absorption spectra governing optoelectronic device performance can be modified with relative ease [7]. Strains permit to change the gap character from direct to indirect one and vice versa. Under tensile strains bilayer $\mathrm{MoS}_{2}$ can retain the indirect gap. Isotropic tensile strains can reduce the band gap of bilayer $\mathrm{MoS}_{2}$ and is more effective in reducing the band gap of $\mathrm{MoS}_{2}$ monolayer than the compressive strains. Compressive strains, in its turn, can reinforce the direct semiconducting character of $\mathrm{MoS}_{2}$ monolayer. Bilayer $\mathrm{MoS}_{2}$ can change from an indirect to a direct semiconductor under sufficiently large compressive strain; and isotropic compressive strain enlarges the band gap of bilayer $\mathrm{MoS}_{2}$. On can note that the strain can reduce the band gap of the $\mathrm{MoS}_{2}$ monolayer regardless of the strain direction [8].

Fusing various TMDs in lateral $\left(\mathrm{MoX}_{2}\right)_{\mathrm{n}} /\left(\mathrm{WX}_{2}\right)_{\mathrm{m}}(\mathrm{X}=\mathrm{S}, \mathrm{Se}, \mathrm{Te})$ type-II band alignment heterostructures may establish a promising class of materials for solar energy harvesting. Such heterostructures can facilitate efficient inplane electron-hole separation for light detection or harvesting with a type II characteristic preserved up to $12 \%$ of uniaxial strain. Uniaxial tensile strains allows to manipulate the electronic and optical properties (density of states, dielectric constant, absorption edge) of those $\mathrm{MoX}_{2} / \mathrm{WX}_{2}(\mathrm{X}=\mathrm{S}, \mathrm{Se}, \mathrm{Te})$ heterostructures in order to maximize solar energy harvesting. It is shown that uniaxial tensile strain of $4 \%$ could increase the power conversion efficiency of the lateral $\left(\mathrm{MoX}_{2}\right)_{\mathrm{n}} /\left(\mathrm{WX}_{2}\right)_{\mathrm{m}}$ heterostructure by about $35 \%$ compared with that of the pristine system [9].

One more way of band-gap engineering is a different orientation of separated layers within the heterostructure. The energy landscape of stacked $\mathrm{MoS}_{2} / \mathrm{MoSe}_{2}$ heterostructure shows very low variations under rotation, with no strongly preferred alignments [10]. Upon stacking $\mathrm{WS}_{2} / \mathrm{WSe}_{2}$ and $\mathrm{MoS}_{2} / \mathrm{WSe}_{2}$ bilayers behave like a direct band gap material. Alternation of individual layers of different TMDs $\left(\mathrm{MoS}_{2}, \mathrm{WS}_{2}, \mathrm{WSe}_{2}\right.$ and $\left.\mathrm{MoSe}_{2}\right)$ thus permits to generate bilayers with a direct band gap ranging from $0.79 \mathrm{eV}$ to $1.16 \mathrm{eV}$ and to fabricate materials with unprecedented optical and physicochemical properties [11].

Also noteworthy, phase engineering of 2D TMDs allows electrical properties from semiconducting to semi-metallic or metallic due to the different symmetry between the common ground state H-phase and T- (or T'-) phases. As an example, T-phase $\mathrm{WTe}_{2}$ may serve as semi-metallic contacts [11b].

\section{Synthesis}

The way of monolayer fabrication is a crucial point for desired properties. Between the main techniques for fabrication of TMDs monolayers one usually distinguish "bottom-up" and "top-down" approaches. Within the first group the individual constituents are pieced together into more complex structures, the second one rely on heteroepitaxy i.e. by cracking of a precursor into its constituents by chemical vapor deposition (CVD)

Micromechanical cleavage with adhesive tape from bulk crystals is a one of top-down methods of fabrication TMDs layers [12]. This technique was developed for graphene. It permits obtaining single-crystal flakes of high purity and cleanliness suitable for fundamental characterization and fabrication of individual devices. However, the method is not scalable and does not allow systematic control of flake thickness and size. For large quantities of exfoliated nanosheets liquid-phase preparation is used. To be exfoliated in a liquid, the layers of TMDs could be intercalated by ionic species. The disadvantage of the method is that the resulting exfoliated material differs structurally and electronically from the bulk material. TMDs can be exfoliated as well by ultrasonication in appropriate liquids (organic solvents, aqueous surfactant solutions, or solutions of polymers in solvents). Ultrasonication results in the mechanical exfoliation of layered crystals to give flakes that are a few hundred nanometers in size [12].

The main advantages of ion exfoliation with $\mathrm{Li}$ intercalation improved by an electrochemical method are high yield of monolayers and more faster and controllable process of exfoliation. But Li compounds are flammable under ambient conditions. Moreover, $\mathrm{Li}$ is an increasingly expensive resource. Liquid exfoliation is insensitive to ambient conditions, but it yields a relatively low concentration of monolayer flakes. Consequently, for electronic or photonic applications where monolayers are required, well-annealed ion-exfoliated nanosheets will be useful. For applications such as composite materials where large quantities are required liquid exfoliation will be preferred.

One between bottom-up methods for synthesizing TMDs is chemical vapor deposition, which allows growing atomically thin films of $\mathrm{MoS}_{2}$ on insulating substrates using different solid precursors heated to high temperatures [12]. The final film thickness is dependent on the concentration or thickness of the initial precursor. However, precise 
control of the number of layers over a large area has not been achieved. Atomic layer deposition (ALD) is an attractive technique because it allows a low temperature deposition by chemical reactions of precursors directly on the surface. It was successfully used to grow $\mathrm{MoSe}_{2}$. However self-limited surface reactions are a vast field of research that requires the development of adequate precursors for other TMDs. Usually island growth mode or formation of clusters rather than continuous films are obtained [12b].

One more way is a chemical preparation of $\mathrm{MoS}_{2}$ and $\mathrm{MoSe}_{2}$ using hydrothermal synthesis that is the growth of single crystals from an aqueous solution in an autoclave at high temperature and pressure [13, 14]. A number of other methods to synthesize $\mathrm{WS}_{2}, \mathrm{MoS}_{2}, \mathrm{WSe}$ and $\mathrm{MoSe}_{2}$ are reported [12, 15-19]. Such methods give reasonably good-quality materials with typical flake sizes of hundreds of nanometers to a few micrometers. But the flake thickness is not conclusively shown to be monolayers.

\section{Electronic structure and related properties}

First-principle calculations show $\mathrm{MoS}_{2}, \mathrm{WS}_{2}, \mathrm{WSe}$, and $\mathrm{MoSe}_{2}$ to be indirect-gap semiconductors [20, 21]. However, some of their single layers already possess a direct gap behavior, which can be used to design new optoelectronic devices and sensors. A unit cell of $2 \mathrm{D} \mathrm{MoS}_{2}$ is shown in Fig. 1. Here the parameter $\mathrm{Z}$ shows the direction of strain which could be applied to the material. In order to model an application of strains the lattice constant of $\mathrm{MoS}_{2}$ was varied with a fixed step, the optimized lattice parameter $a$ was $3.18 \AA$, the minimum value of $Z$ is $3.13 \AA$. Uniaxial tension along the $z$ axis leads to the shift of the conduction band minimum and increase of the first direct gap (Fig. 2). The decrease of $Z$ parameter to $3.06 \AA$ leads to a decrease of the band gap. Band gap behavior of strained 2D $\mathrm{MoS}_{2}$ upon variation of $a$ lattice parameter and $Z$ parameter is presented in Fig. 3. One conclude that it is possible to find a region with an appropriate band gap value and behavior of first transition, which will be applicable for straindependent optoelectronic devices.

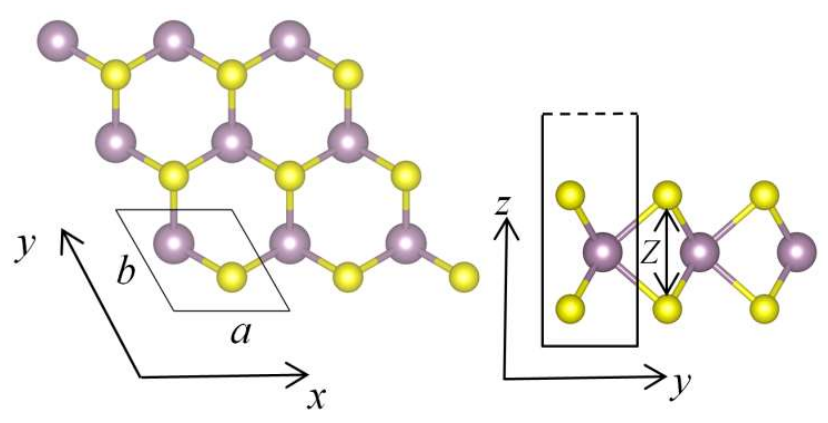

Fig $1.1 \times 1 \times 1$ unit cell structure of $2 \mathrm{D} \mathrm{MoS}_{2}$. Big balls are Mo atoms, small balls are $\mathrm{S}$ atoms
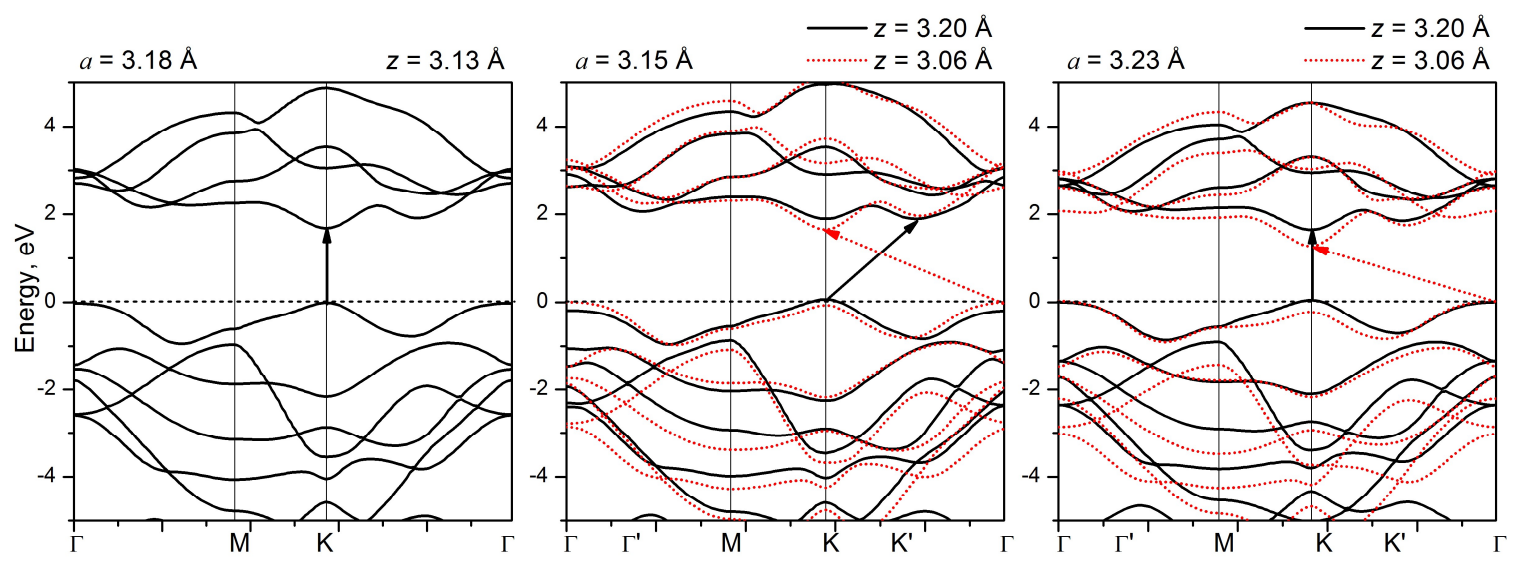

Fig. 2. Band structure of strained 2D MoS 2 . 


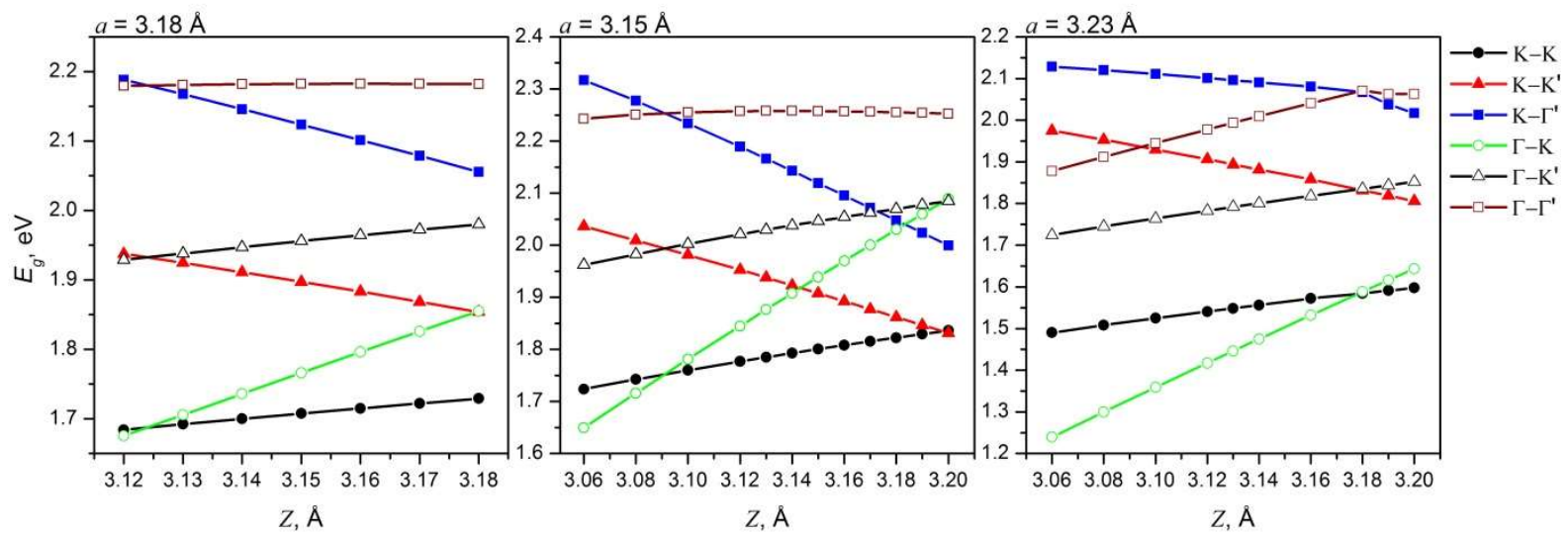

Fig. 3. Band gap behavior of strained 2D $\mathrm{MoS}_{2}$.

Band gap modifications in $2 \mathrm{D} \mathrm{MoS}_{2}, \mathrm{MoSe}_{2}, \mathrm{WS}_{2}$, and $\mathrm{WSe}_{2}$ in dependence on the presence of vacancies or impurities in the positions of chalcogen atoms, namely, oxygen atoms adsorbed on the surface or embedded in the lattice, are shown in Fig. 4. It can be seen that the band gaps of TMDs are strongly changed by vacancies, and in a less extent by replacement of chalcogen atoms by smaller oxygen atoms and adsorption of oxygen atoms. The replacement of chalcogen atoms with oxygen ones leads to an increase of the band gap, while the adsorption of oxygen on the surface leads to a slight decrease in the band gap, which can be used for precise control of the electronic, optical and catalytic properties of such 2D crystals [21].

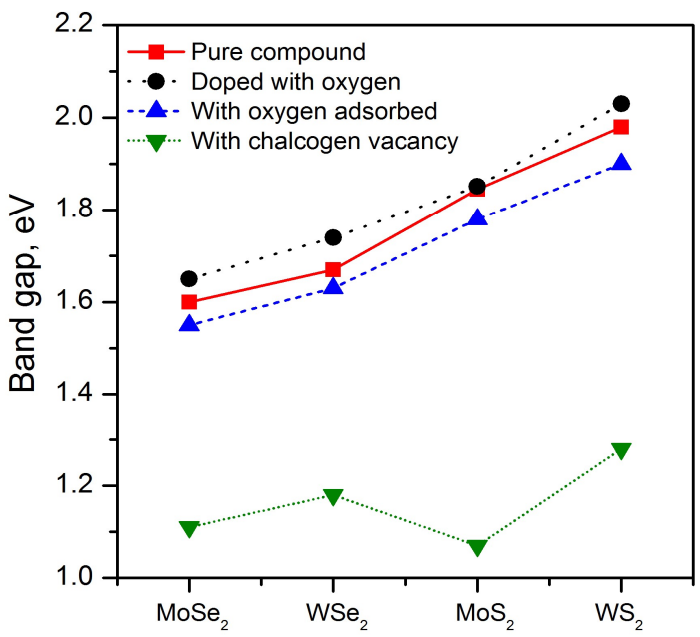

Fig. 4. Band gaps in 2D dichalcogenides depending on point defects.

Substitution of sulphur atoms in $\mathrm{MoS}_{2}$ compound by isovalent tellurium ones reveals that monolayer $\mathrm{MoS}_{2-x} \mathrm{Te}_{x}$ remains a direct-gap semiconductor for small concentrations of $\mathrm{S}$ or $\mathrm{Te}(x<0.5$ or $x>1.5)$, while it changes its character to indirect-gap for $x=0.5-1.5$. Band gap of a ternary semiconductor $\mathrm{MoS}_{2-x} \mathrm{Te}_{x}$ can be adjusted by means of an appropriate choice of the concentration ratio between the $\mathrm{S}$ and Te atoms. The dependence of the gap value on the concentration of Te atoms is shown in Fig. 5 [22]. Thus, the band gap engineering is possible as well by introducing an isovalent impurity. 


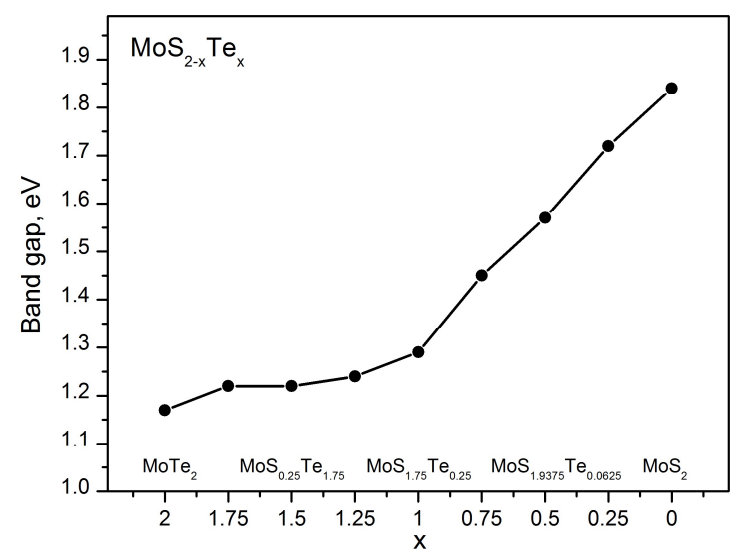

Fig. 5. Energy band gap of one monolayer $\mathrm{MoS}_{2-\mathrm{x}} \mathrm{Te}_{\mathrm{x}}$.

\section{Applications}

Layers of TMDs due to the tunable band gap (but not solely) can be used for fabrication of a wide range of optoelectronic devices such as field effect transistors, memories, solar cells, light-emitting diodes, photodetectors, , photoelectrochemical and photocatalytic cells, or spintronic devices. A $\mathrm{MoS}_{2}$ thin film double-layer transistor fabricated with ion gel gate dielectrics on a plastic substrate was reported to exhibit excellent band transport with a low threshold voltage, high mobility and a high on/off current ratio [23]. It is characterized by remarkably high mechanical flexibility, and absence of degradation of electrical characteristics upon significant bending. On the base of $\mathrm{MoS}_{2}$ /graphene heterostructure a memory cell with a single-layer $\mathrm{MoS}_{2}$ semiconducting channel, graphene contacts and multilayer graphene (MLG) floating gate is proposed [24]. An ambipolar field-effect transistor is constructed on exfoliated thin flakes of $\mathrm{WS}_{2}$ with an ionic liquid dielectric [25].

Top-gate phototransistors based on single-, double-, and triple-layer $\mathrm{MoS}_{2}$ nanosheets were fabricated for photovoltaics [26]. Phototransistors with triple $\mathrm{MoS}_{2}$ layers exhibited excellent photodetection capabilities for red light, while those with single- and double-layers turned out to be quite useful for green light detection. The varied functionalities are attributed to energy gap modulation by the number of $\mathrm{MoS}_{2}$ layers.

\section{Heterostructures}

Recall that combination of the layers of different TMDs gives a possibility to obtain a direct-gap hybrid 2D crystals. They could be synthesized either by sandwiching exfoliated monolayers using a careful mechanical transfer or by means of a layer-by-layer CVD [27] or ALD [12b] technique. $\mathrm{MoS}_{2}$ can be randomly alloyed with $\mathrm{MoSe}_{2}$ to form ternary compounds by the thermal vapor deposition (TVD) method for bandgap engineering of the $\mathrm{MoS}_{2}$-based 2D materials. Large single-crystal atomic layers of $\mathrm{MoS}_{2}$ combined with $\mathrm{WS}_{2}$ ones can be epitaxially grown by sequence to create vertical or lateral 2D heterostructures [28]. Alternating separate layers of different TMDs, such as $\mathrm{MoS}_{2}$, $\mathrm{WS}_{2}, \mathrm{WSe}_{2}$, and $\mathrm{MoSe}_{2}$, with a certain orientation relative to each other make it possible to form double layers with a direct energy transition in the range from $0.79 \mathrm{eV}$ to $1.16 \mathrm{eV}[11]$.

In order to determine electronic properties of such systems, an impact of the stacking arrangement as well as vacancies and substitution of atoms were analyzed for $\mathrm{MoS}_{2} / \mathrm{MoSe}_{2}, \mathrm{MoS}_{2} / \mathrm{WS}_{2}, \mathrm{WS}_{2} / \mathrm{WSe}_{2}$, and $\mathrm{MoSe}_{2} / \mathrm{WSe}_{2}$ heterostructures [29]. Several variants of two-layer heterostructures with different arrangement of layers were analyzed e.g.: when layers are arranged symmetrically relative to each other (A-stacking), and arrangement of layers with placement, similar to the arrangement of layers in a bulk material (B-stacking). $\mathrm{MoS}_{2} / \mathrm{MoSe}_{2}, \mathrm{MoS}_{2} / \mathrm{WS}_{2}$, $\mathrm{WS}_{2} / \mathrm{WSe}_{2}$, and $\mathrm{MoSe}_{2} / \mathrm{WSe}_{2}$ layer combinations were considered. Te atoms substituted one chalcogen atoms in each monolayer or Te atoms and one vacancy in different monolayers. Resulting dependencies are shown in Fig. 6. 


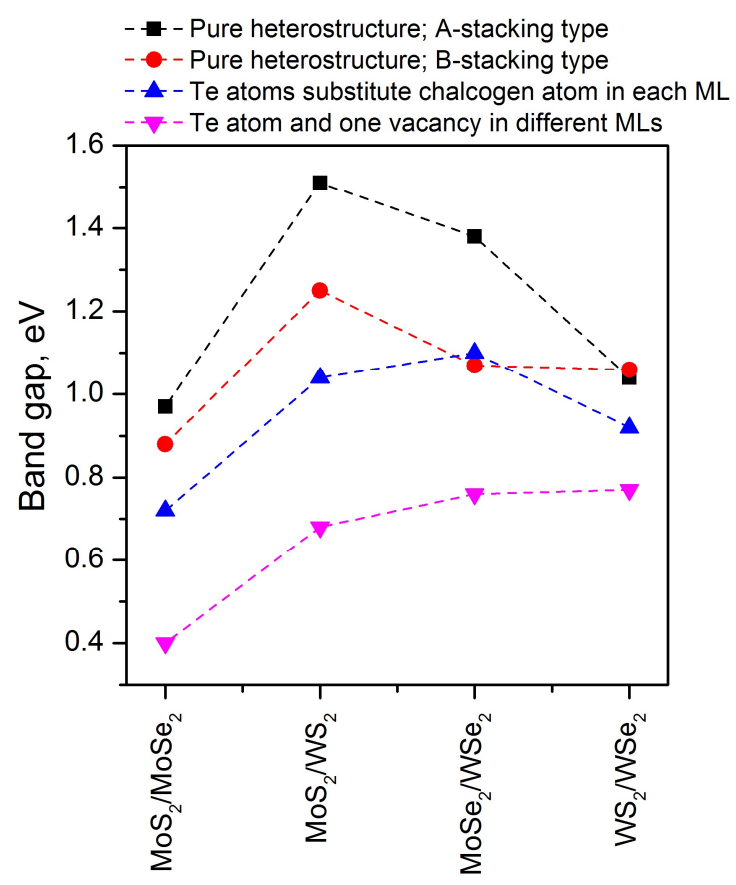

Fig. 6. The band gaps in the $\mathrm{MoS}_{2} / \mathrm{MoSe}_{2}, \mathrm{MoS}_{2} / \mathrm{WS}_{2}, \mathrm{WS}_{2} / \mathrm{WSe}_{2}, \mathrm{MoSe}_{2} / \mathrm{WSe}_{2}$ heterostructures depending on the arrangement of layers and defects.

Magnetic moments of the systems were studied when Mn atoms replace Mo atoms either in one of the layers, or in both layers. It has been found that the magnetic moment on the manganese atom is the highest when the impurity is placed in both layers, and the mutual arrangement of the layers does not play a significant role in this case (Fig. 7).

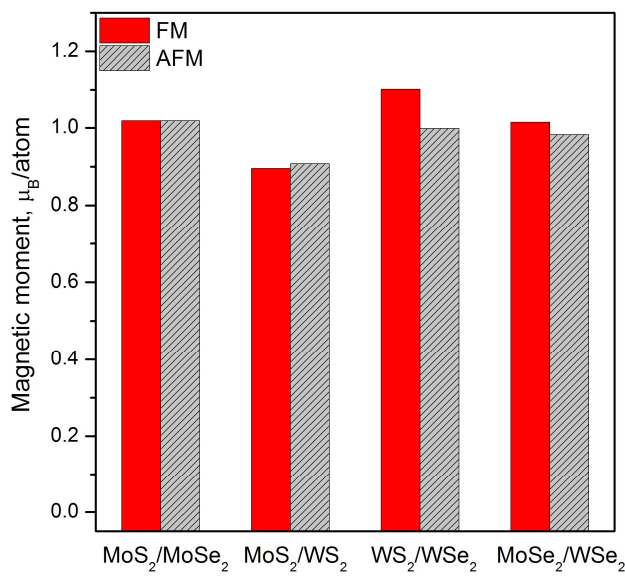

a)

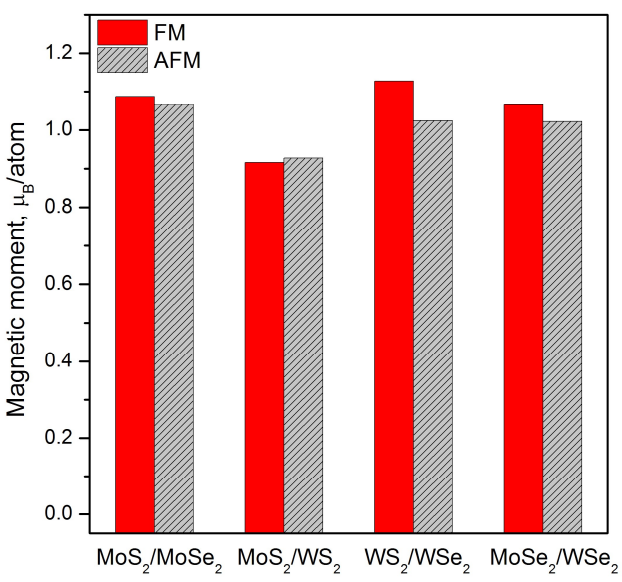

b)

Fig. 7. Magnetic moments per impurity atoms: a) A-stacking type arrangement of the layers; b) B-stacking type. Impurity atoms are located in both layers.

Mn atoms in Mo and W sites lead to magnetic ordering with a high spin polarization [30]. Electronic spin-polarized band structures of the systems are presented in Fig. 8. Spin-polarized electronic band spectra for $\mathrm{MoS}_{2} / \mathrm{MoSe}_{2}$, $\mathrm{MoS}_{2} / \mathrm{WS}_{2}, \mathrm{WS}_{2} / \mathrm{WSe}_{2}, \mathrm{MoSe}_{2} / \mathrm{WSe}_{2}$ heterostructures with $\mathrm{Mn}$ atoms in place of Mo or $\mathrm{W}$ atoms for the mirror arrangement of layers are shown in Fig. 8a, and for layers with displacement in Fig. 8b, with one impurity atom in the lower layer and the second one in the upper layer. $\mathrm{WS}_{2} / \mathrm{WSe}_{2}$ with A-stacking demonstrates metallic properties, while in three other variants $\left(\mathrm{MoS}_{2} / \mathrm{MoSe}_{2}, \mathrm{MoS}_{2} / \mathrm{S}_{2}\right.$ and $\left.\mathrm{MoSe}_{2} / \mathrm{Se}_{2}\right)$ the electronic states at the Fermi level in one spin 
channel only indicate the half-metallic nature. The B-type stacking leads to the half-metallic properties for all of the presented heterostructures [30].
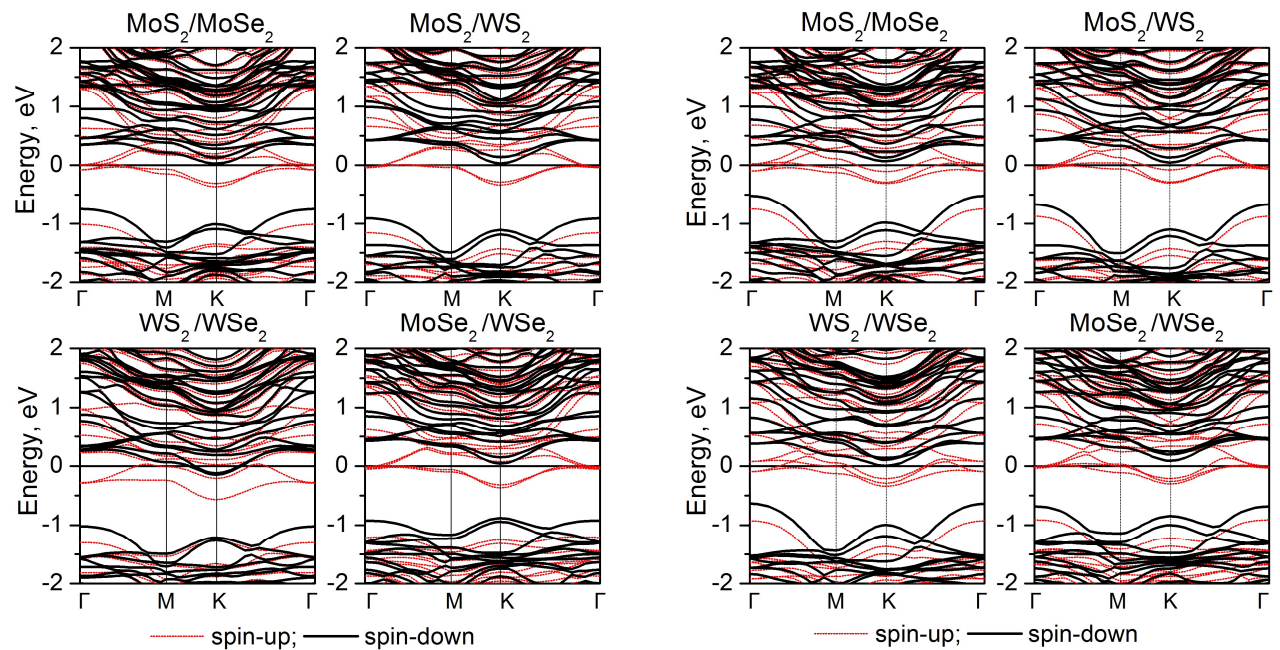

Fig. 8. Electronic spin-polarized band structures of Mn-doped layered two-dimensional crystals $\mathrm{MoS}_{2} / \mathrm{MoSe}_{2}, \mathrm{MoS}_{2} / \mathrm{WS}_{2}, \mathrm{WS}_{2} / \mathrm{WSe}_{2}$, $\mathrm{MoSe}_{2} / \mathrm{WSe}_{2}$ in the case of A-stacking type (left) and B-stacking type (right).

An effect of substituting position on electronic properties of $\mathrm{WS}_{2} / \mathrm{WSe}_{2}$ heterostructures containing Te atoms was investigated for the case of impurity in the position of chalcogen or metal atoms (Fig. 9). An investigation of substitution of S or Se atoms by Te has shown the preservation of semiconductor properties of the structure. The first gap changes its nature from direct to indirect one depending on the position of substituting atoms and the distance between them. The heterostructure with Te atoms in the positions of Se ones preserves a direct gap, while substitution of $\mathrm{S}$ atoms led preferably to an indirect-gap behavior. When Te substituted both $\mathrm{S}$ and Se atoms band gap changes in a different way depending on the mutual location of the Te atoms. Such possibility of direct/indirect gap switching opens the prospects for band-gap engineering and creation of multilayered structures with tuned gap, extremely important for development of novel electronic and photonic devices [31].

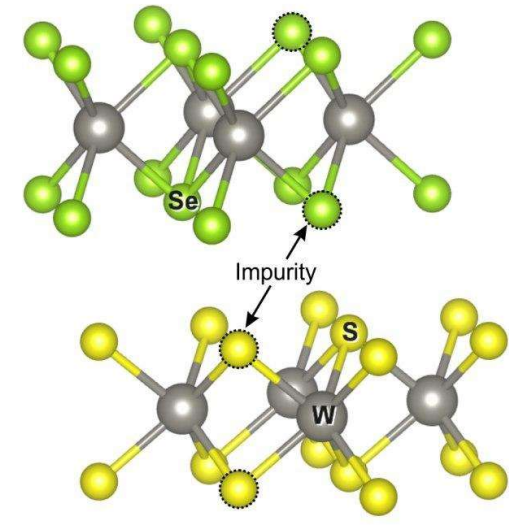

a)

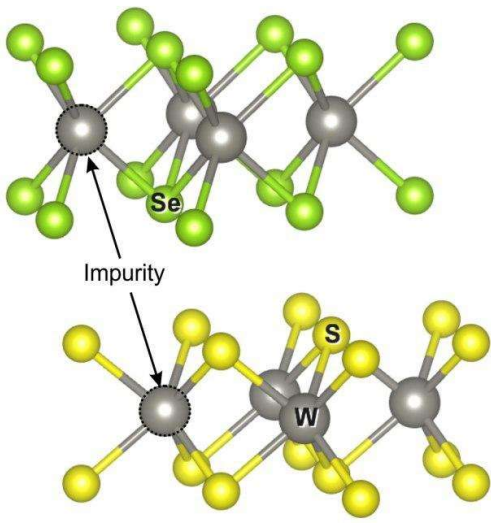

b)

Fig. 9. $\mathrm{WS}_{2} / \mathrm{WSe}_{2}$ heterostructure: Te atoms replace $\mathrm{S}$ atoms, Se atoms, or both $\mathrm{S}$ and $\mathrm{Se}$ atoms (a); Te atoms replace $\mathrm{W}$ atoms.

Substitution of $\mathrm{W}$ sites in $\mathrm{WS}_{2}$ layer reduces the gap. Te atoms both in $\mathrm{WS}_{2}$ and $\mathrm{WSe}_{2}$ layers reduce the gap, while the structure still exhibits semiconductor behavior. Te atoms in $\mathrm{W}$ sites in $\mathrm{WSe}_{2}$ layer produce new states at the Fermi level giving rise to metallic properties [32]. 


\section{Conclusion}

In this short review, we have used the results of our ab initio modeling to the possible ways to change and control the properties of 2D TMDs, essentially the bandgap, magnetic moment and spin polarizations. It is shown that nonmagnetic TMDs can get magnetic moments upon strains, doping, adsorption of non-magnetic atoms, and presence of zigzag edges in nanoribbons. Heterostructures with different stacking of the monolayers could be fabricated on the base of semiconductor direct-gap monolayers. Such bilayers in contrast to the pristine homo-material possess direct band gaps. Electronic structure of each layer is tunable and permits to fabricate materials with unprecedented properties. Band gap value of TMDs can be controlled by means of doping, strains, and a combination of monolayers, thus opening the possibility of creation transistors, light-emitting diodes, photoelectrochemical cells, photovoltaic and optoelectronic devices on their base.

\section{Acknowledgements}

The work was performed in the framework of State Scientific Program "Functional and Engineering Materials, Nanomaterials". The authors are grateful to Goethe-Institüt for financial support within the MOST project for enhancing professional contacts between Belarus and the EU which made participation in NANOTEXNOLOGY 2020 conference possible. V. E. Borisenko acknowledges the partial financial support of the "Improving of the Competitiveness" Program of the National Research Nuclear University MEPhI - Moscow Engineering Physics Institute.

\section{References}

[1] M. Ye, D. Zhang, Y. K. Yap, Electronics 6 (2017) 43(1-39).

[2] P. Tao, H. Guo, T. Yang, Z. Zhang, J. Appl. Phys. 115 (2014) 054305(1-5).

[3] S. Tongay, S. S. Varnoosfaderani, B. R. Appleton, J. Wu, A. F. Hebard, Appl. Phys. Lett. 101 (2012) $123105(1-4)$

[4] J. He, K. Wu, R. Sa, Q. Li, Y. Wei, Appl. Phys. Lett. 96 (2010) 082504(1-3).

[5] Y. Li, Z. Zhou, S. Zhang, Z. Chen, J. Am. Chem. Soc. 9(49) (2008) 16739-16744.

[6] A. Ramasubramaniam, D. Naveh, Physical Review B 87 (2013) 19520 (1-7).

[7] H. J. Conley et al., Nano Lett 13(8) (2013) 3626-3630.

[8] P. Lu et al., Phys. Chem. Chem. Phys. 14, 13035-13040 (2012).

[9] J. Lee, J. Huang, B. G. Sumpter, M. Yoon, 2D Mater. 4 (2017) 021016 (1-8).

[10] G. C. Constantinescu, N. D. M. Hine, Phys. Rev. B 91 (2015) 195416(1-9).

[11]H. Terrones, F. Lopez-Urias, M. Terrones, Scientific Reports 3 (2013) 1549(1-7).

[12] Q. H. Wang, K. Kalantar-Zadeh, A. Kis, J. N. Coleman, M. S. Strano, Nature nanotechnology 7 (2012) 699-712.

[13] Y. Peng et al., J. Solid State Chem. 159 (2001) 170-173.

[14] Y. Peng et al., Chem. Lett. 30 (2001) 772-773.

[15]H. S. S. Ramakrishna Matte et al., Angew. Chem. Int. Ed. 49 (2010) 4059-4062.

[16]H. S. S. Ramakrishna Matte, R. B. Plowman., R. Datta, C. N. R. Rao, Dalton Trans. 40 (2011) 1032210325.

[17] J. N. Coleman et al., Science 331 (2011) 568-571.

[18] Y. Shi et al., Nano Lett. 12 (2012) 2784-2791.

[19] Y. Li, J. Am. Chem. Soc. 133 (2011) 7296-7299.

[20] A. Krivosheeva, V. Shaposhnikov, V. Borisenko, J-L. Lazzari, C. Waileong, J. Gusakova, B.K. Tay, J. Semicond. 36(12) (2015) 122002(1-6).

[21] A.V. Krivosheeva, V.L. Shaposhnikov, V.E. Borisenko, J.-L. Lazzari, N.V. Skorodumova, B.K. Tay, Int. J. Nanotechnol. 12(8/9) (2015) 654-662.

[22] A. V. Krivosheeva, V.L. Shaposhnikov, V.E. Borisenko, Doklady BSUIR 4(98) (2016) 98-101- in Russian.

[23] J. Pu et al., Nano Lett. 12 (2012) 4013-4017.

[24] S. Bertolazzi, D. Krasnozhon, A. Kis, ACS Nano 7 (4) (2013) 3246-3252.

[25] D. Braga, I. Gutiérrez Lezama, H. Berger, A.F. Morpurgo, Nano Lett. 12(10) (2012) 5218-5223.

[26] H. S. Lee et al., Nano Lett. 12 (2012) 3695-3700.

[27] A. K. Geim, I. V. Grigorieva, Nature 499 (2013) 419-425. 
[28] H. F. Liu, Dr. S. L. Wong, D. Z. Chi, Chem. Vap. Deposition 21 (2015) 241-259.

[29] V. L. Shaposhnikov, A. V. Krivosheeva, V. E. Borisenko, Phys. Status Solidi B 256 (2019) 1800355 (1-7).

[30] A. V. Krivosheeva, V. L. Shaposhnikov, Vestnik of the Foundation for Fundamental Research 80 (2017) 106-112 - in Russian.

[31] A. V. Krivosheeva, V. L. Shaposhnikov, V. E. Borisenko, J.-L. Lazzari, Journal of Materials Science 55(23) (2020) 9695-9702.

[32] A. V. Krivosheeva, V. L. Shaposhnikov, V. E. Borisenko, J.-L. Lazzari, International Journal of Nanoscience, 18(3-4) (2019) 1940007(1-4). 